\title{
Corrigendum: New Form of Kane's Equations of Motion for Constrained Systems
}

\author{
Carlos M. Roithmayr* \\ NASA Langley Research Center, Hampton, Virginia 23681 \\ Abdulrahman H. Bajodah ${ }^{\dagger}$ \\ King Abdulaziz University, Jeddah, Saudi Arabia 21589 \\ Dewey H. Hodges; and Ye-Hwa Chen ${ }^{\S}$ \\ Georgia Institute of Technology, Atlanta, Georgia 30332
}

\section{Introduction}

A correction to the previously published article "New Form of Kane's Equations of Motion for Constrained Systems" "1 is presented. Misuse of the transformation matrix between time rates of change of the generalized coordinates and generalized speeds (sometimes called motion variables) resulted in a false conclusion concerning the symmetry of the generalized inertia matrix. The generalized inertia matrix (sometimes referred to as the mass matrix) is in fact symmetric and usually positive definite when one forms nonminimal Kane's equations for holonomic or simple nonholonomic systems, systems subject to nonlinear nonholonomic constraints, and holonomic or simple nonholonomic systems subject to impulsive constraints according to Refs. 1, 2, and 3 , respectively. The mass matrix is of course symmetric when one forms minimal equations for holonomic or simple nonholonomic systems using Kane's method as set forth in Ref. 4.

*Aerospace Engineer, Space Mission Analysis Branch, Mail Stop 462, E-mail: c.m.roithmayr@larc.nasa.gov. Senior Member, AIAA.

${ }^{\dagger}$ Assistant Professor, Aeronautical Engineering Department, E-mail: abajodah@kau.edu.sa.

${ }^{\ddagger}$ Professor, School of Aerospace Engineering, E-mail: dewey.hodges@ae.gatech.edu. Fellow, AIAA.

$\S$ Professor, George W. Woodruff School of Mechanical Engineering, E-mail: yehwa.chen@me.gatech.edu. 


\section{Symmetry of the Generalized Inertia Matrix}

Let $\mathcal{S}$ be a simple nonholonomic dynamical system, and let $\mathcal{R}$ be an inertial frame of reference in which the configuration of $\mathcal{S}$ is described by a set of $n$ generalized coordinates $q_{1}, \ldots, q_{n}$. The velocity of a generic particle $P$ of the system relative to $\mathcal{R}$ can be written as

$$
{ }^{\mathcal{R}} \mathbf{v}^{P}=\sum_{r=1}^{n}{ }^{\mathcal{R}} \mathbf{v}_{r}^{P}(q, t) u_{r}+{ }^{\mathcal{R}} \mathbf{v}_{t}^{P}(q, t)
$$

where the generalized speeds $u_{1}, \ldots, u_{n}$ are scalar variables satisfying some nonholonomic constraint relations linear in $u_{1}, \ldots, u_{n}$, and ${ }^{\mathcal{R}} \mathbf{v}_{1}^{P}, \ldots,{ }^{\mathcal{R}} \mathbf{v}_{n}^{P}$ are the corresponding holonomic partial velocities of the system. The generalized speeds also satisfy the kinematical differential equations

$$
\dot{q}=C(q, t) u+D(q, t)
$$

The system's kinetic energy relative to $\mathcal{R}$ is given by ${ }^{1}$

$$
K=\frac{1}{2} u^{T} M(q, t) u+N(q, t) u+R(q, t)
$$

where $M \in \mathbb{R}^{n \times n}$ is symmetric, $N \in \mathbb{R}^{1 \times n}$, and $R \in \mathbb{R}$. In most cases $M$ is positive definite; it can be nonnegative definite (positive semidefinite) when, for example, one neglects a central principal moment of inertia of a rigid body belonging to $\mathcal{S}$, such as a slender rod, or when one uses a particle with no mass to represent a point whose motion must be known. The $r$ th nonholonomic generalized inertia force $\tilde{F}_{r}^{\star}$ can be written in terms of the holonomic generalized inertia forces $F_{r}^{\star}$ and in terms of $K$ according to Eqs. (4.11.4) and (5.6.6) respectively of Ref. 4,

$$
\begin{aligned}
\tilde{F}_{r}^{\star} & =F_{r}^{\star}+\sum_{s=1}^{n-p} F_{p+s}^{\star} A_{s r} \\
& =-\sum_{s=1}^{n}\left[\frac{d}{d t}\left(\frac{\partial K}{\partial \dot{q}_{s}}\right)-\frac{\partial K}{\partial q_{s}}\right]\left(W_{s r}+\sum_{k=1}^{n-p} W_{s, p+k} A_{k r}\right) \quad(r=1, \ldots, p)
\end{aligned}
$$

where the elements of $W$ have the same meanings as in Eqs. (2.14.5) of Ref. 4,

$$
\dot{q}=W u+X
$$

The matrix $W$ is thus in fact identical to $C(q, t)$; it is wrongly taken to be $C^{-1}(q, t)$ following Eq. (27) in Ref. 1, leading to errors in Eqs. (31), (32), (35), and (36), and to the incorrect conclusion that the generalized inertia matrix $Q$ can be asymmetric. A rectification of the errors is provided in what follows. 
The observation that generalized inertia forces are linear in $\dot{u}$ is made in Ref. 1 and will be revisited shortly. Consequently $F^{\star}$, a column matrix whose elements are $F_{r}^{\star}$, can be written in the form

$$
F^{\star}=-Q(q, t) \dot{u}-L(q, u, t)
$$

In Ref. 1 the nature of matrix $Q$ is considered by using the kinetic energy of the system. Upon appealing to Eqs. (3) and (5) it can be seen that

$$
\frac{\partial K}{\partial \dot{q}}=\frac{\partial K}{\partial u} \frac{\partial u}{\partial \dot{q}}=\left[u^{T} M+N\right] W^{-1}
$$

Therefore, defining the matrix $A_{2}$ as in Eq. (16) of Ref. 1

$$
A_{2}=\left[\begin{array}{ll}
I & A^{T}
\end{array}\right]
$$

yields the following matrix representation of Eqs. (4)

$$
\begin{aligned}
A_{2} F^{\star} & =-A_{2} W^{T}\left(\frac{d}{d t}\left[W^{-T} M u+W^{-T} N^{T}\right]-K_{q}^{T}\right) \\
& =-A_{2} W^{T}\left(W^{-T} M \dot{u}+\frac{d}{d t}\left[W^{-T} M\right] u+\frac{d}{d t}\left[W^{-T} N^{T}\right]-K_{q}^{T}\right)
\end{aligned}
$$

where

$$
K_{q}=\left\lfloor K_{q_{1}} \ldots K_{q_{n}}\right\rfloor=\frac{\partial K}{\partial q}
$$

If Eq. (6) is multiplied by $A_{2}$ and compared with Eq. (9), we obtain

$$
\begin{aligned}
& Q \triangleq W^{T} W^{-T} M=M \\
& L \triangleq W^{T}\left(\frac{d}{d t}\left[W^{-T} M\right] u+\frac{d}{d t}\left[W^{-T} N^{T}\right]-K_{q}^{T}\right)
\end{aligned}
$$

Equations (11) and (12) correct Eqs. (35) and (36) of Ref. 1. The foregoing analysis shows that the generalized inertia matrix $Q$ is symmetric and, as noted previously, usually positive definite. In addition to its role in Ref. 1, $Q$ plays a central part in forming nonminimal Kane's equations for systems subject to the constraints treated in Refs. 2 and 3.

\section{Alternative Demonstration of Symmetry}

It is noted in Ref. 4 that frequently it is inefficient to use $K$ to construct $F_{r}^{\star}$ or $\tilde{F}_{r}^{\star}$; likewise, readers of Ref. 1 are made aware that the use of $K$ to form $Q$ or $L$ is undesirable. The definition of $F_{r}^{\star}$ given in Eqs. (4.11.2) and (4.11.3) of Ref. 4 provides alternative means for showing that $Q$ 
is identical to $M$, and for constructing $M$ and $L$. When $\mathcal{S}$ is made up of particles $P_{1}, \ldots, P_{\nu}$,

$$
F_{r}^{\star} \triangleq-\sum_{i=1}^{\nu} m_{i}{ }^{\mathcal{R}} \mathbf{v}_{r}^{P_{i}} \cdot{ }^{\mathcal{R}} \mathbf{a}^{P_{i}} \quad(r=1, \ldots, n)
$$

where $m_{i}$ is the mass of $P_{i}$, and where the acceleration of $P_{i}$ in $\mathcal{R}$, denoted by ${ }^{\mathcal{R}} \mathbf{a}^{P_{i}}$, can be written in terms of partial velocities by differentiating Eq. (1) with respect to $t$ in $\mathcal{R}$ as shown in Refs. 5 and 2 .

$$
\begin{aligned}
{ }^{\mathcal{R}} \mathbf{a}^{P_{i}} & =\sum_{r=1}^{n}\left[{ }^{\mathcal{R}} \mathbf{v}_{r}^{P_{i}} \dot{u}_{r}+\left(\frac{{ }^{\mathcal{R}}}{d t} \mathcal{R}^{\mathcal{R}} \mathbf{v}_{r}^{P_{i}}\right) u_{r}\right]+{ }^{\mathcal{R}} \frac{d}{d t} \mathcal{R} \mathbf{v}_{t}^{P_{i}} \\
& =\sum_{r=1}^{n}{ }^{\mathcal{R}} \mathbf{v}_{r}^{P_{i}} \dot{u}_{r}+{ }^{\mathcal{R}} \mathbf{a}_{t}^{P_{i}} \quad(i=1, \ldots, \nu)
\end{aligned}
$$

where the remainder term of the acceleration is defined as

$$
{ }_{\mathcal{R}} \mathbf{a}_{t}{ }^{P_{i}} \triangleq \sum_{r=1}^{n}\left(\frac{\mathcal{R}}{d t}^{\mathcal{R}} \mathbf{v}_{r}^{P_{i}}\right) u_{r}+\frac{}{\mathcal{R}}_{d t} \mathcal{R}^{\mathcal{R}} \mathbf{v}_{t}^{P_{i}} \quad(i=1, \ldots, \nu)
$$

Substitution from Eqs. (14) into (13) yields

$$
\begin{aligned}
F_{r}^{\star} & =-\sum_{i=1}^{\nu} m_{i}{ }^{\mathcal{R}} \mathbf{v}_{r}^{P_{i}} \cdot\left(\sum_{s=1}^{n}{ }^{\mathcal{R}} \mathbf{v}_{s}{ }^{P_{i}} \dot{u}_{s}+{ }^{\mathcal{R}} \mathbf{a}_{t}{ }^{P_{i}}\right) \\
& =-\sum_{s=1}^{n}\left(\sum_{i=1}^{\nu} m_{i}{ }^{\mathcal{R}} \mathbf{v}_{r}^{P_{i}} \cdot{ }^{\mathcal{R}} \mathbf{v}_{s}^{P_{i}}\right) \dot{u}_{s}-\sum_{i=1}^{\nu} m_{i}{ }^{\mathcal{R}} \mathbf{v}_{r}{ }^{P_{i}} \cdot{ }^{\mathcal{R}} \mathbf{a}_{t}{ }^{P_{i}} \quad(r=1, \ldots, n)
\end{aligned}
$$

It is immediately clear that $F_{r}^{\star}$ is linear in $\dot{u}_{1}, \ldots, \dot{u}_{n}$; each time derivative of a generalized speed is multiplied by an inertia coefficient defined as

$$
m_{r s} \triangleq \sum_{i=1}^{\nu} m_{i}{ }^{\mathcal{R}} \mathbf{v}_{r}^{P_{i}} \cdot{ }^{\mathcal{R}} \mathbf{v}_{s}^{P_{i}}=m_{s r} \quad(r, s=1, \ldots, n)
$$

A matrix $M$ whose elements are $m_{r s}$ is referred to as a generalized inertia matrix or mass matrix. Taken together, Eqs. (16) and (17) show conclusively that $M$ is symmetric by definition when obtained with Kane's method as set forth in Ref. 4. Although the present demonstration involves a holonomic system, the result is readily shown to apply also to a simple nonholonomic system; the interested reader is referred to Eqs. (5.5.3) and (5.5.4), and Problem 10.11 in Ref. 4. The form of $m_{r s}$ is dependent on one's choice of generalized speeds because the partial velocities are dependent on this choice; however, $M$ is symmetric for any valid choice whatsoever. The matrix $M$ is in fact identical to $Q$ that appears in Eq. (6) and thus a symmetric mass matrix appears in the nonminimal 
Kane's equations presented in Refs. 1, 2, and 3. The property of symmetry does not necessarily extend to a mass matrix obtained with other variants of Kane's method, such as the lower triangular matrix obtained with the method presented in Ref. 6 .

It is important to note that not all methods of deriving dynamical equations guarantee a symmetric mass matrix. For example, it is well known that Newton-Euler methods do not guarantee symmetry. Although Lagrange's equations always produce a symmetric mass matrix, the same cannot be said in general for their cousins, the Boltzmann-Hamel equations. ${ }^{7}$

As indicated on p. 150 of Ref. 4, $M$ is the matrix in Eq. (3) associated with the portion $K_{2}$ of kinetic energy that is quadratic in the generalized speeds

$$
K_{2}=\frac{1}{2} u^{T} M u=\frac{1}{2} \sum_{r=1}^{n} \sum_{s=1}^{n} m_{r s} u_{r} u_{s}
$$

A comparison of Eqs. (16) and (6) reveals that the elements of matrix $L$ are given by $L_{r} \triangleq$ $\sum_{i=1}^{\nu} m_{i}{ }^{\mathcal{R}} \mathbf{v}_{r}^{P_{i}} \cdot{ }^{\mathcal{R}} \mathbf{a}_{t}^{P_{i}}(r=1, \ldots, n)$. As pointed out in Ref. 1 , it is advisable to construct $F_{r}^{\star}$ according to the procedure set forth in Sec. 4.11 of Ref. 4 rather than by forming the matrices $M$ and $L$.

\section{References}

${ }^{1}$ Bajodah, A. H., Hodges, D. H., and Chen, Y.-H., “A New Form of Kane's Equations of Motion for Constrained Systems," Journal of Guidance, Control, and Dynamics, Vol. 26, No. 1, 2003, pp. 79-88.

${ }^{2}$ Bajodah, A. H., Hodges, D. H., and Chen, Y.-H., "Nonminimal Kane's Equations of Motion for Multibody Dynamical Systems Subject to Nonlinear Nonholonomic Constraints," Multibody System Dynamics, Vol. 14, No. 2, 2005, pp. 155-187.

${ }^{3}$ Bajodah, A. H., Hodges, D. H., and Chen, Y.-H., "Nonminimal Generalized Kane's Impulse-Momentum Relations," Journal of Guidance, Control, and Dynamics, Vol. 27, No. 6, 2004, pp. 1088-1092.

${ }^{4}$ Kane, T. R. and Levinson, D. A., Dynamics: Theory and Applications, McGraw-Hill Book Company, New York, 1985, pp. 46, 124-129, 150, 151, 153, 319.

${ }^{5}$ Rosenthal, D. E., “Order n Formulation for Robotic Systems,” The Journal of the Astronautical Sciences, Vol. 38, No. 4, 1990, pp. 511-529.

${ }^{6}$ Rosenthal, D. E., "Triangularization of Equations of Motion for Robotic Systems," Journal of Guidance, Control, and Dynamics, Vol. 11, No. 3, 1988, pp. 278-281.

${ }^{7}$ Meirovitch, L., Methods of Analytical Dynamics, McGraw-Hill Book Company, New York, 1970, pp. $162-163$. 